\title{
$\underline{\text { Re: A response to Willis et al. }}$
}

\section{Authors: L Anquinet, K Raus, S Sterckx, T Smets, L Deliens, JAC Rietjens}

To the editor:

We wish to thank Dr. Willis and colleagues for their interest in our study on similarities and differences between continuous sedation until death and euthanasia as perceived by Belgian professional caregivers (1). We would like to take the opportunity to respond to their comments.

Dr. Willis et al's principal concern with our paper is that we are not clear about what is meant by terminal sedation either for them, as the study group, or for the members of the focus groups. We share Dr. Willis' view that there is a need for a clear-cut and widely agreed upon term and definition for the practice of sedation at the end of life.

However, in our focus group study, we aimed to gain more insight into all possible types of sedation used by professional caregivers at the end of life, as well as to obtain a deeper understanding of one particular type of sedation used in end-of-life care: continuous sedation until death. We believe that this broad and descriptive definition of sedation is far less confusing than terms like 'terminal sedation' (which was used by Dr. Willis but not by us), a term which is particularly subject to debate because it may provoke different connotations among different people (2).

Another concern with our paper of Dr. Willis and colleagues is that we do not distinguish correctly between the practice of continuous sedation until death and euthanasia. As explained in the paper, it was not the principal focus of our study to investigate this distinction. However, it turned out to be one of the main themes that surfaced in the focus group discussions. As we also describe in our paper, and as Dr. Willis and colleagues correctly stressed, sedation guidelines state that in a correct use of continuous sedation until death the drugs should be titrated until symptom control is achieved and should not be aimed at hastening death, and in that way sedation is said to be different from the practice of euthanasia. However, our study shows that in practice, -although in many cases this distinction is clearly experienced by professional caregivers- they sometimes experience difficulties distinguishing the use of sedation from the practice of euthanasia. We feel this is an important result of our study, because it shows that theoretical distinctions are sometimes more complex and not always tenable in clinical practice. We support Dr. Willis and colleagues' call for the importance of unambiguous and sophisticated symptom relief at the end of life. Our study adds to this call by showing the huge complexities involved. Understanding these complexities is an important aspect of improving end-of-life care as well. 


\section{References}

(1) Anquinet L, Raus, K, Sterckx S, Smets T, Deliens L, Rietjens JAC. Similarities and differences between continuous sedation until death and euthanasia - professional caregivers' attitudes and experiences: a focus group study. Palliat Med 2013;27:553-561.

(2) Sterckx S. Raus K. Mortier F. (Eds) Continuous sedation at the end of life - Ethical, Clinical and Legal Perspectives. Cambridge: CambridgeUniversity Press: 2013. 